\title{
SOME ARITHMETIC PROPERTIES OF GENERALIZED BERNOULLI NUMBERS
}

BY L. CARLITZ

Communicated by Gerald B. Huff, November 8, 1958

In a recent paper [2] Leopoldt has defined generalized Bernoulli numbers and polynomials in the following manner. Let $f$ be a fixed integer $\geqq 1$ and $\chi(r)$ a primitive character $(\bmod f)$. Put

$$
\sum_{r=1}^{f} \chi(r) \frac{t e^{(r+u) t}}{e^{f t}-1}=\sum_{n=0}^{\infty} B_{\chi}^{n}(u) \frac{t^{n}}{n !}, \quad B_{\chi}^{n}=B_{\chi}^{n}(0) .
$$

For $f=1, \chi$ is the principal character and $B_{\chi}^{n}$ reduces to the ordinary Bernoulli number $B_{n}$. The main result of Leopoldt's paper is an analog of the Staudt-Clausen theorem.

In the present paper we obtain the following theorems, the first of which is a refinement of Leopoldt's analog of the Staudt-Clausen theorem. We assume $f>1$.

Theorem 1. If $f$ is divisible by at least two different primes, then $B_{x}^{n} / n$ is an algebraic integer. If $f=p, p>2, B_{x}^{n} / n$ is an algebraic integer unless

$$
\mathfrak{p}=(p, 1-\chi(g)) \neq(1),
$$

in which case

$$
p B_{\chi}^{n} \equiv p-1\left(\bmod p^{n+1}\right) ;
$$

if $f=p^{\mu}, p>2, \mu>1, B_{x}^{n} / n$ is integral unless

$$
\mathfrak{B}=\left(p, 1-\chi(g) g^{n}\right) \neq(1),
$$

in which case

$$
(1-\chi(1+p)) \frac{B_{\chi}^{n}}{n} \equiv 1(\bmod \mathfrak{P}) ;
$$

$g$ is a primitive root $\left(\bmod p^{r}\right)$ for all $r \geqq 1$. If $f=4$, then

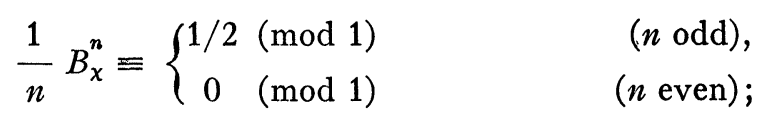

if $f=2^{\mu}, \mu>2$, then $B_{x}^{n} / n$ is integral.

Theorem 2. If $f=p^{\mu}$, then 


$$
\sum_{s=0}^{r}(-1)^{r-s} \frac{B_{\chi}^{n+1+s w}}{n+1+s w} \equiv 0\left(\bmod \left(q^{n}, q^{e r}\right)\right),
$$

where $q$ is a prime $\neq p$ and $q^{e-1}(q-1) \mid w$. If $f \neq p^{\mu}$, then (4.8) holds for arbitrary primes $q$.

TheOREM 3. If $p$ is a prime such that $p \nmid f, p^{e-1}(p-1) \mid m$, then

$$
\frac{1}{m+1} B_{\chi}^{m+1} \equiv \frac{1}{f}(1-\chi(p)) \sum_{s=1}^{f} s \chi(s)\left(\bmod p^{e}\right) \text {. }
$$

In particular, if $\chi(p)=1$ or $\chi(-1)=1$, then

$$
\frac{1}{m+1} B^{m+1} \equiv 0\left(\bmod p^{e}\right)
$$

In particular, for $f=4$, Theorem 3 reduces to the following known result for the Euler numbers:

$$
E_{m} \equiv \begin{cases}0\left(\bmod p^{e}\right), & p \equiv 1(\bmod 4), \\ 2\left(\bmod p^{e}\right), & p \equiv 3(\bmod 4),\end{cases}
$$

where $p^{e-1}(p-1) \mid m$.

The proof of these theorems makes use of various known properties of the ordinary Bernoulli numbers as well as the Eulerian numbers defined by [1]

$$
\frac{1-\lambda}{e^{t}-\lambda}=\sum_{n=0}^{\infty} H_{n}(\lambda) \frac{t^{n}}{n !} .
$$

In particular we cite the representation

$$
\frac{1}{n+1} B_{\chi}^{n+1}=\frac{\tau(\chi)}{f} \sum_{r=1}^{f} \frac{\bar{\chi}(r) \alpha^{r}}{1-\alpha^{r}} H_{n}(\alpha)
$$

where

$$
\tau(\chi)=\sum_{r=1}^{f} \chi(r) \alpha^{r}, \quad \alpha=e^{2 \pi i / f}
$$

\section{REFERENCES}

1. G. Frobenius, Über die Bernoulli'schen Zahlen und die Euler'schen Polynome, Preuss. Akad. Wiss. Sitzungsber. (1910) pp. 809-847.

2. H. W. Leopoldt, Eine Verallgemeinerung der Bernoullischen Zahlen, Abh. Math. Sem. Univ. Hamburg vol. 22 (1958) pp. 131-140.

DUKE UNIVERSITY 